\title{
Transitionsforschung in der Schweiz - ein kurzer Überblick
}

\author{
Kurt Häfeli, Markus P. Neuenschwander \& Stephan Schumann
}

Am Übergang von der Schule in den Beruf werden wichtige Weichen gestellt, die den Lebenslauf eines Menschen vorbereiten. Jugendliche müssen sich zwischen verschiedenen Ausbildungsgängen und Berufen entscheiden und sich für gewünschte Zulassungen qualifizieren. Dabei unterscheiden sich die Anforderungen im Bildungssystem deutlich von denjenigen im Wirtschaftssystem bzw. in der Arbeit. Entsprechend sind Schulen mit der Aufgabe konfrontiert, einerseits die eigenen bildungsimmanenten Ansprüche zu erfüllen, andererseits die Schülerinnen und Schüler auf den neuen beruflichen Kontext vorzubereiten. Die Betriebe stehen vor der Herausforderung, durch Produktivität ihr Überleben bzw. ihren Gewinn zu sichern und gleichzeitig ihren eigenen Nachwuchs zu qualifizieren und eine hohe Ausbildungsqualität bereitzustellen.

Entsprechend anforderungsreich gestaltet sich dieser Übergangsprozess für die jungen Menschen und deren Unterstützungspersonen. Daher ist es nicht erstaunlich, dass das Interesse an diesen Übergangsprozessen in der öffentlichen Wahrnehmung stark zugenommen hat. Nicht nur die Berufs- und Laufbahnberatung, sondern auch die Schule und Ausbildungsinstitutionen sowie die Betriebe suchen Wege, wie junge Menschen beim Berufseintritt unterstützt werden können, so dass jede Person zur richtigen beruflichen Funktion findet. Gleichzeitig sind der Übergang in den Beruf und die berufliche Entwicklung häufig von Unterbrüchen und Umwegen charakterisiert. Frühe berufliche Ziele werden immer seltener direkt erreicht. Aufgrund des Fachkräftemangels hat sich der Handlungsbedarf zugespitzt. Damit sind auch spätere Phasen der beruflichen Entwicklung (Berufswechsel, Nachholbildung, Weiterbildung etc.) relevant geworden. 


\section{$1 \quad$ Einbettung und Zielsetzung}

Trotz der herausragenden Bedeutung des Übergangs von der Schule in den Beruf und der weiteren beruflichen Entwicklung für Individuen und Betriebe ist die Forschungslage in diesem Themenbereich erstaunlich mager. In einer Literaturübersicht konnten Häfeli und Schellenberg (2009) zwar knapp 60 Studien oder Projektevaluationen zum Übergang von der Schule in die Arbeitswelt zusammenstellen, die in der Schweiz von 2000-2009 durchgeführt wurden. Allerdings waren viele dieser Untersuchungen in ihren Aussagen begrenzt, weil sie nur einige wenige Merkmale untersuchten, sich auf Stichproben mit eingeschränkter Aussagekraft bezogen oder ein querschnittliches Design aufwiesen. Zudem konzentrierten sie sich meist auf die erste Schwelle, d.h. den Übergang von der Sekundarstufe I in die Sekundarstufe II, in zweiter Linie auf die Berufsausbildung, wobei die wichtigen Lehrvertragsauflösungen selten untersucht wurden. Wenig Beachtung fand die zweite Schwelle - der Übergang von der Sekundarstufe II in die weiterführende Tertiärstufe oder in den Arbeitsmarkt.

Einige wenige Studien waren breit angelegt und als Längsschnitt konzipiert: TREE (Bertschy, Böni, \& Meyer, 2007), FASE B (Neuenschwander, Frey, \& Gasser, 2007), INTSEP (Haeberlin, Imdorf, \& Kronig, 2004), LEVA (Schmid \& Stalder, 2008), Hochbegabung (Stamm, 2005, 2007), COCON (Buchmann, 2007), ZLSE (Spiess Huldi, 2009). Einige dieser Studien sind in der Zwischenzeit abgeschlossen (Eckhart, Haeberlin, Sahli Lozano, \& Blanc, 2011; Neuenschwander, Gerber, Frank, \& Rottermann, 2012; Schmid, 2010), andere wurden fortgesetzt (Bergman, Hupka-Brunner, Keller, Meyer, \& Stalder, 2011; Buchmann, 2013; Buchmann \& Kriesi, 2012; Schellenberg, Häfeli, Schmaeh, \& Hättich, 2013). Eine bilanzierende Gesamtschau daraus zu ziehen, ist schwierig, da die Studien zu unterschiedlich angelegt sind. Es wird aber doch klar, dass die soziale Herkunft, der Schultyp der Sekundarstufe I und die schulische Leistungsfähigkeit, aber auch das Geschlecht und die Nationalität bzw. der Migrationshintergrund eine wichtige Rolle spielen (Häfeli \& Schellenberg, 2009; Neuenschwander, 2014). Der Erfolg im Bildungswesen scheint also wesentlich von sozialstrukturellen Merkmalen abhängig zu sein. Insgesamt zeigen diese Befunde, dass berufliche Kompetenzentwicklung und Berufserfolg als Produkt vielfältiger Einflusssysteme (Person, Schule, Beruf, Familie) verstanden werden müssen und sich nicht auf wenige Einflussbedingungen oder -systeme reduzieren lassen. Zur Erklärung dieser Befunde werden je nach Studie unterschiedliche theoretische Konzepte verwendet, die das Zusammenspiel von Person und Umwelt thematisieren. Diese unterschiedlichen konzeptuellen Zugänge sind zum einen hilfreich für eine möglichst perspektivenreiche 
Erschliessung des Gegenstands, zum anderen sind sie häufig nur schwer miteinander zu verknüpfen.

Trotz diesen Studien bleiben weiterhin bedeutsame Lücken, auch wenn in der Zwischenzeit neue Projekte durchgeführt wurden (etwa zur zweijährigen Grundbildung mit Attest (Hofmann \& Häfeli, 2012; Kammermann \& Hättich, 2010) oder zu Übertritten, Korrekturen und Verläufen während der Berufslehre (Berweger, Krattenmacher, Salzmann, \& Schönenberger, 2013).

Aufgrund dieser Ausgangslage und angesichts der Aktualität und praktischen Relevanz des Themas hat sich das Staatssekretariat für Bildung, Forschung und Innovation (SBFI) entschieden, der Transitionsforschung höhere Aufmerksamkeit zukommen zu lassen und verschiedene Forschungsvorhaben zu finanzieren. Damit sollen Ergebnisse generiert werden, die für die öffentliche Steuerung der Transitionsprozesse genutzt werden können. Zwischenzeitlich liegen erste Ergebnisse der erstaunlich vielfältigen Transitionsforschung in der Schweiz vor. Diese Projekte entwickelten nicht nur wichtige theoretische Grundlagen, sondern auch empirische Evidenzen für die Analyse und Steuerung bedeutsamer Passagen im Lebenslauf. Die Projekte sind in verschiedenen Disziplinen wie Psychologie, Soziologie, Erziehungswissenschaften oder Bildungsökonomie verortet. Dadurch ergeben sich vielfältige Perspektiven auf die Übergänge und Entscheidungsprozesse an der ersten Schwelle (Sekundarstufe I - II), während der Berufsausbildung und an der zweiten Schwelle (Sekundarstufe II - Tertiärstufe oder Arbeitsmarkt) sowie im weiteren Berufsverlauf.

Für den vorliegenden Band wurden die sieben Projekte, welche im Zeitraum 2010-2014 vom SBFI im Rahmen der Transitionsforschung gefördert wurden, eingeladen, einen Originalbeitrag zu verfassen. Zusätzlich wurden von den Herausgebern - ohne Anspruch auf Vollständigkeit - vier weitere Projekte ausgewählt und die Projektleitungen angefragt, zentrale und aktuelle Ergebnisse zur beruflichen Laufbahn aus ihren Studien zu berichten, wobei bei diesen zusätzlichen Projekten ein spezieller Fokus auf die bisher untervertretene französischsprachige Schweiz gelegt wurde.

Damit liegen nun erstmals ausgewählte Ergebnisse dieser elf Forschungsprojekte in einem Band vor. Es dürfte sichtbar werden, dass diese schweizerischen Projekte einen wichtigen Beitrag leisten können, um Transitionsprozesse in diesen zentralen bildungs- und berufsbiographischen Phasen besser zu verstehen. 


\section{Skizze des schweizerischen Berufsbildungssystems}

Um die Beiträge des vorliegenden Bandes besser einordnen zu können, soll nachfolgend die schweizerische Berufsbildung kurz vorgestellt werden.

Die Berufsbildung umfasst die berufliche Grundbildung auf Sekundarstufe II sowie die höhere Berufsbildung auf Tertiärstufe. Die berufliche Grundbildung wird in der Regel im Anschluss an die obligatorische Schulzeit durchlaufen, aber rund ein Sechstel aller Jugendlichen schlagen Umwege wie Zwischenlösungen oder Brückenangebote ein. Die berufliche Grundbildung umfasst folgende Bildungsangebote (vgl. Abbildung 1):

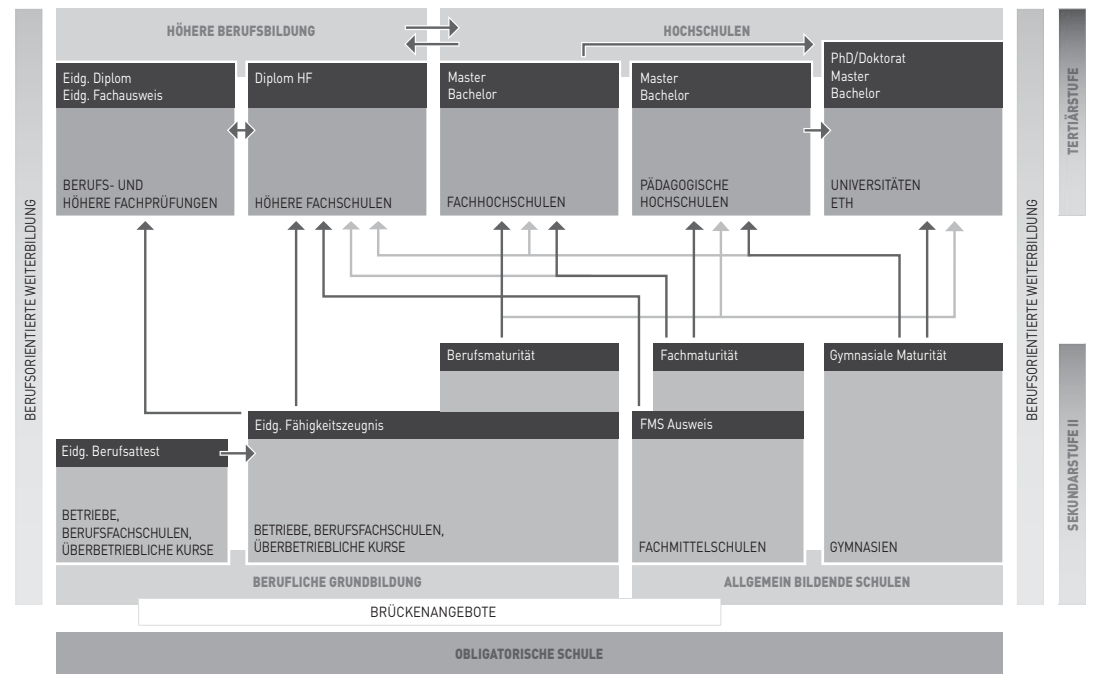

Abbildung 1 Skizze des schweizerischen Berufsbildungssystems (Quelle: Factsheet Berufliche Grundbildung, SBFI, 2014)

- Die 3- oder 4-jährige berufliche Grundbildung mit eidgenössischem Fähigkeitszeugnis (EFZ) dient der Vermittlung der Qualifikationen zur Ausübung eines bestimmten Berufes und öffnet den Zugang zur höheren Berufsbildung.

- Die 2-jährige berufliche Grundbildung mit eidgenössischem Berufsattest (EBA) erlaubt vorwiegend praktisch begabten Jugendlichen die Erlangung eines anerkannten Abschlusses, welcher Zugang zu weiterführenden Bildungsangeboten bietet. 
- Die eidgenössische Berufsmaturität ergänzt die berufliche Grundbildung EFZ im Sinne einer vertieften Allgemeinbildung. Sie ermöglicht leistungsstarken Jugendlichen den prüfungsfreien fachgebundenen Zugang zu einer Fachhochschule. Mit einer Ergänzungsprüfung ist auch der Zugang über eine Passarelle an eine Schweizer Universität oder eine der Eidgenössischen Technischen Hochschulen möglich.

Anders als in vielen anderen europäischen Ländern ist in der Schweiz zwar auch eine gewisse Akademisierungstendenz zu verzeichnen, allerdings ist die berufliche Grundbildung weiterhin die meistgewählte Erstausbildung. Zwei Drittel der Jugendlichen entscheiden sich für eine solche berufliche Grundbildung. Nach dieser Grundbildung sind vielfältige Weiterbildungen möglich. Neben der bereits erwähnten Fachhochschule (Zugang mit einer Berufsmaturität) können im Bereich der Höheren Berufsbildung eine durch die Berufsverbände organisierte Berufsprüfung (z.B. Baupolier), eine Höhere Fachprüfung (z.B. Schreinermeister, dipl. Finanzexpertin) oder eine Höhere Fachschule (Pflegefachmann/-frau HF) absolviert werden.

Die berufliche Grundbildung erfolgt zum überwiegenden Teil dual, im Lehrbetrieb und in der Berufsfachschule. Die Lernenden sind dabei aktiv in betriebliche Prozesse integriert und erlernen im Betrieb berufspraktische Kenntnisse und Fähigkeiten. Die Berufsfachschule vermittelt die schulische Bildung und bietet zusätzlich den Berufsmaturitätsunterricht an. Alternativ kann die berufliche Grundbildung auch vollständig im schulischen Umfeld, z.B. in einer Lehrwerkstätte oder Handelsmittelschule, stattfinden.

\section{$3 \quad$ Gliederung des Buchs}

Das vorliegende Buch berücksichtigt in Übereinstimmung mit den vielfältigen Facetten, die mit dem Übergang von der Schule in den Beruf aufscheinen, theoretische Ansätze aus verschiedenen Disziplinen wie der Erziehungswissenschaft, Psychologie, Soziologie und der Ökonomie. Während manche Artikel aus einer eher entscheidungstheoretischen Perspektive analysieren (zum Beispiel Rational Choice Theorie, Humankapitaltheorie, motivationspsychologische Theorien), wie Ausbildungswege und berufliche Kontexte gewählt werden, verfolgen andere Texte einen eher sozialisationstheoretischen Ansatz und beschreiben, wie sich junge Menschen beim Eintritt in neue Ausbildungs- und Berufskontexten mit Erwartungen und Normen auseinandersetzen, neue soziale Beziehungen eingehen und eine Rolle finden. In Folge der interdisziplinären Zugänge stehen in gewissen 
Artikeln eher die institutionelle Steuerung bzw. Unterstützung der Bildungswege bzw. Berufskarriere im Vordergrund, während in anderen Artikeln eher der aktive junge Mensch im Zentrum steht, der seinen Weg plant. Allerdings wird in allen Beiträgen explizit oder implizit eine Konzeption des Verhältnisses oder der Passung zwischen der Institution bzw. des Kontextes und des Individuums und seinen Ressourcen betrachtet. Berufliche Passagen werden immer vom Individuum vollzogen, aber sie werden von steuernden Institutionen und gesellschaftlichen Normen vorstrukturiert.

Vor diesem Hintergrund gliedert sich das Buch in drei Teile, welche verschiedene Phasen der beruflichen Entwicklung betreffen: 1) die Phase der Berufsfindung und des Einstiegs in die Berufsausbildung, 2) die Phase während der Berufsausbildung und 3) die Phase der Eingliederung in den Beruf bzw. der Erwerbstätigkeit und der beruflichen Laufbahn.

Im ersten Teil befassen sich Becker und Glauser mit geschlechtsspezifischen Berufswünschen und Ausbildungsentscheidungen. Mittels Paneldaten wird für Jugendliche der Deutschschweiz gezeigt, dass der sozioökonomische Status des Elternhauses, das damit einhergehende Motiv des intergenerationalen Statuserhalts sowie der Lebenslaufplanungen dazu beitragen können, die Segregation der Berufswahl nach der Geschlechtszugehörigkeit zu erklären. Beim anschliessenden Übergang von der Schule in die berufliche Grundbildung müssen sich die Jugendlichen einer sich verändernden sozialen Situation anpassen. Nägele und Neuenschwander zeigen auf, dass dies besser gelingt, wenn Jugendliche einen Lehrberuf gewählt haben, den sie als passend zu ihren Interessen und Fähigkeiten wahrnehmen. Die wahrgenommene Passung ist ein Ergebnis des Berufswahlprozesses und kann sich zu Beginn der beruflichen Grundbildung aufgrund neuer Erfahrungen verändern.

Der zweite Teil widmet sich spezifischen Prozessen und Verläufen während der Berufsausbildung. Eine grosse Herausforderung stellen dabei Lehrvertragsauflösungen dar: Rund jeder vierte Lehrvertrag wird vorzeitig aufgelöst, besonders betroffen davon sind der Koch- und der Malerberuf. Negrini, Forsblom, Schumann und Gurtner untersuchen die Rolle der Lehrbetriebe in diesen Berufen und fragen, ob eine hohe Ausbildungsqualität eine Art Schutzfaktor gegen Lehrvertragsauflösungen darstellen könnte. Die Perspektive der Jugendlichen wird in einer qualitativen Längsschnittstudie von Lamamra und Duc im Kanton Waadt untersucht. Dabei werden die vielfältigen und keineswegs immer geradlinigen Ausbildungswege nach einer Lehrvertragsauflösung aufgezeigt. Meist führen sie wieder zurück in die Berufsbildung, manchmal aber auch zu einem Lehrabbruch. Mit der anderen Seite der Medaille, dem Ausbildungserfolg, befassen sich Schafer und Baeriswyl. Sie analysieren Einflussfaktoren auf die Abschlussnote bei der kauf- 
männischen Berufslehre, der mit Abstand häufigsten beruflichen Grundbildung in der Schweiz. Im letzten Beitrag dieses Teils beleuchten Rastoldo und Mouad die spezifische Situation im Kanton Genf. Die duale Berufsbildung ist dort - wie in der gesamten französischsprachigen Schweiz - weniger stark verankert als in der Deutschschweiz. Sie wird oft erst auf Umwegen über Brückenangebote oder allgemeinbildende Schulen erreicht. Dies führt zu markant später im Bildungsverlauf erworbenen Abschlüssen als theoretisch möglich wäre.

Im dritten und umfangreichsten Teil zur weiteren beruflichen Laufbahn wird zunächst die zweijährige Grundbildung mit eidgenössischem Berufsattest (EBA) von Hofmann und Häfeli untersucht. Mit der Einführung dieser Ausbildungsform sollten die Arbeitsmarktfähigkeit und die Durchlässigkeit zu weiterführenden Ausbildungen im Vergleich mit der ehemaligen Anlehre verbessert werden. Im Zentrum des Beitrags steht die Frage, ob sich das Ausbildungsgefäss sowohl für Lernende aus Regelklassen wie aus Sonderklassen eignet und wie sich die jeweiligen beruflichen Laufbahnen nach Abschluss weiterentwickeln. Eine weitere Phase der beruflichen Laufbahn wird im Beitrag von Hupka-Brunner, Scharenberg, Meyer und Müller untersucht. Welchen beruflichen Status haben junge Erwachsene in der Schweiz zehn Jahre nach Beendigung der obligatorischen Schule, im Alter von durchschnittlich 26 Jahren erreicht - und welche Faktoren beeinflussen sie? Mit Analysen der TREE-Daten weisen sie einerseits auf eine vergleichsweise hohe Status-"Vererblichkeit" zwischen den Generationen hin, andererseits auf erhebliche Verletzungen des meritokratischen Prinzips, wonach für den Bildungserfolg und die erreichte Position in der Gesellschaft vor allem die individuelle Leistung massgeblich sein sollte. Im Beitrag von Düggeli und Neuenschwander werden drei unterschiedliche berufsbiografische Entscheidungssituationen verglichen: der Eintritt in die duale Berufsbildung, der Austritt aus dieser sowie die Weiterbildungsorientierung von Erwerbstätigen, drei bis sechs Jahre nach dem Lehrabschluss. Die Autoren zeigen auf, dass Merkmale der Entscheidungsfindung in allen drei Situationen gleicherweise mit der wahrgenommenen Passung zusammenhängen. Zur Erreichung eines tertiären Bildungsabschlusses steht den Jugendlichen in der Schweiz eine Vielzahl an Bildungspfaden zur Auswahl. Im Beitrag von Pfister und Tuor Sartore wird analysiert, was die Wahl eines rein akademischen, eines rein beruflichen oder eines sogenannten gemischten, d.h. berufliche und akademische Ausbildungsinhalte umfassenden Bildungspfades determiniert. Die empirischen Resultate, basierend auf dem Mikrozensus Ausund Weiterbildung 2011, zeigen, dass bei rein beruflichen und rein akademischen Bildungspfaden ein systematischer Zusammenhang mit den Bildungsabschlüssen der Eltern besteht, aber nicht so bei gemischten Bildungspfaden. Schliesslich untersuchen Schellenberg, Schmaeh, Häfeli und Hättich, wie Laufbahnen von der 
ersten beruflichen Entscheidung mit 15 Jahren bis zum 49. Lebensjahr verlaufen. Dabei wird zum einen die horizontale und vertikale Mobilität untersucht und zum anderen danach gefragt, welche Merkmale der Person und des Umfeldes sich auf den späteren Berufsstatus auswirken. Die Ergebnisse zeigen, dass berufliche Mobilität bis in die Lebensmitte zwar vorkommt, jedoch in einem eingeschränkteren Ausmass als oft angenommen wird. Mit zunehmendem Alter werden Wechsel seltener und die berufliche Kontinuität nimmt über den Laufbahnverlauf zu.

Mit den vorliegenden Beiträgen kann erstmals in der Breite aufgezeigt werden, wie die Berufsbildung in der Schweiz an ihren entscheidenden Übergängen im Zusammenspiel von individuellen Faktoren und von Kontextbedingungen funktioniert und welche Herausforderungen für die Zukunft erkennbar sind. Insbesondere die Schlussfolgerungen in den einzelnen Beiträgen zeigen Möglichkeiten der Weiterentwicklung der Berufsbildung auf.

Das Buch richtet sich an Wissenschaftler/innen und Studierende, Fachleute in der Berufsbildung, Berufsberatung, Leitende aus Berufsfachschulen, Organisationen der Arbeitswelt, Verwaltung und Bildungspolitik. Die Beiträge haben einen wissenschaftlichen Anspruch, doch sind sie gut lesbar verfasst und enthalten Schlussfolgerungen für die Gestaltung von Transitionen im Lebenslauf. Auch wenn sich die Projekte auf die Schweiz beziehen, so sind die vielfältigen Befunde häufig grundsätzlicher Art und damit auch für andere Länder relevant.

Abschliessend sei dem SBFI herzlich für die grosszügige finanzielle Unterstützung gedankt, ohne die das Zustandekommen des vorliegenden Buches in dieser Form nicht möglich gewesen wäre.

\section{Literatur}

Bergman, M. M., Hupka-Brunner, S., Keller, A., Meyer, T., \& Stalder, B. E. (Eds.). (2011). Transitionen im Jugendalter. Ergebnisse der Schweizer Längsschnittstudie TREE. Zürich: Seismo.

Bertschy, K., Böni, E., \& Meyer, T. (2007). An der zweiten Schwelle: Junge Menschen im Übergang zwischen Ausbildung und Arbeitsmarkt. Ergebnisübersicht des Jugendlängsschnitts TREE, Update 2007 (pp. 36). Bern: TREE.

Berweger, S., Krattenmacher, S., Salzmann, P., \& Schönenberger, S. (2013). LiSA - Lernende im Spannungsfeld von Ausbildungserwartungen, Ausbildungsrealität und erfolgreicher Erstausbildung. St. Gallen: Pädagogische Hochschule.

Buchmann, M. (2007). Kinder- und Jugendsurvey COCON. Schweizerischer Nationalfonds, Datenauswertung, Zusatzantrag. Zürich.

Buchmann, M. (2013). Bildungsungleichheiten als Gesellschaftliche Herausforderung in der Schweiz In R. Becker, P. Bühler \& T. Bühler (Eds.), Bildungsungleichheit und Gerech- 
tigkeit: Wissenschaftliche und Gesellschaftliche Herausforderungen, (pp. 53-70). Bern: Haupt.

Buchmann, M., \& Kriesi, I. (2012). Geschlechtstypische Berufswahl Jugendlicher. Begabungszuschreibungen, Aspirationen und Institutionen. Kölner Zeitschrift für Soziologie und Sozialpsychologie (Sonderheft), 256-280.

Eckhart, M., Haeberlin, U., Sahli Lozano, C., \& Blanc, P. (2011). Langzeitwirkungen der schulischen Integration. Bern: Haupt.

Haeberlin, U., Imdorf, C., \& Kronig, W. (2004). Von der Schule in die Berufslehre. Bern: Haupt.

Häfeli, K., \& Schellenberg, C. (2009). Erfolgsfaktoren in der Berufsausbildung bei gefährdeten Jugendlichen. Bern: EDK.

Hofmann, C., \& Häfeli, K. (2012). Subjektiver Laufbahnerfolg bei Leistungsschwächeren in einer Berufsausbildung. Schweizerische Zeitschrift für Bildungswissenschaften, 34(1), 117-138.

Kammermann, M., \& Hättich, A. (2010). Mit Berufsattest in den Arbeitsmarkt. BWP(5), 11-14.

Neuenschwander, M. P. (Ed.). (2014). Selektion in Schule und Arbeitsmarkt. Zürich/Chur: Rüegger.

Neuenschwander, M. P., Frey, M., \& Gasser, L. (2007). Familiäre Bedingungen von Schülerleistungen. Schlussbericht zum SNF-Forschungsprojekt Nr. 100013-107733 (pp. 122). Zürich: Jacobs Center for Productive Youth Development, Universität Zürich.

Neuenschwander, M. P., Gerber, M., Frank, N., \& Rottermann, B. (2012). Schule und Beruf. Wege in die Erwerbstätigkeit. Wiesbaden: VS Verlag.

Schellenberg, C., Häfeli, K., Schmaeh, N., \& Hättich, A. (2013). Auswirkungen von erschwerten Startchancen auf den beruflichen Erfolg im mittleren Erwachsenenalter: ein Längsschnitt über 34 Jahre. Schweizerische Zeitschrift für Heilpädagogik, 19(11-12), 26-35.

Schmid, E. (2010). Kritisches Lebensereignis „Lehrvertragsauflösung“. Bern: Hep Verlag.

Schmid, E., \& Stalder, B. E. (2008). Lehrvertragsauflösung: Chancen und Risiken für den weiteren Ausbildungsweg. Ergebnisse aus dem Projekt LEVA (pp. 93). Bern: Bildungsplanung und Evaluation der Erziehungsdirektion des Kantons Bern.

Spiess Huldi, C. (2009). Erfolg im Beruf. Zum Einfluss von Persönlichkeit und psychosozialem Umfeld auf die berufliche Entwicklung Jugendlicher. Zürich/Chur: Rüegger.

Stamm, M. (2005). Zwischen Excellenz und Versagen. Frühleser und Frührechnerinnen werden erwachsen. Zürich: Rüegger.

Stamm, M. (2007). Kluge Köpfe, goldene Hände : ̈̈berdurchschnittlich begabte Lehrlinge in der Berufsbildung. Zürich Rüegger.

Dieses Buch wird unter der Creative Commons Namensnennung-Nicht kommerziell 4.0 International Lizenz (http://creativecommons.org/licenses/by-nc/4.0/deed.de) veröffentlicht, welche für nicht kommerzielle Zwecke die Nutzung, Vervielfältigung, Bearbeitung, Verbreitung und Wiedergabe in jeglichem Medium und Format erlaubt, sofern Sie den/die ursprünglichen Autor(en) und die Quelle ordnungsgemäß nennen, einen Link zur Creative Commons Lizenz beifügen und angegeben, ob Änderungen vorgenommen wurden. 